\title{
HIV-PDI: A Protein Drug Interaction Resource for Structural Analyses of HIV Drug Resistance: 2. Examples of Use and Proof-of-Concept
}

\author{
GHEMTIO Leo $^{1 *}$, SOUCHET Michel ${ }^{2}$, DJIKENG Appolinaire ${ }^{3}$, KEMINSE Lionel ${ }^{2,4}$, KELBERT Patricia $^{1}$, Ritchie David W' ${ }^{1}$, MAIGRET Bernard ${ }^{1,2 *}$ and OUWE-MISSI-
} OUKEM-BOYER Odile ${ }^{4 *}$

${ }^{1}$ Nancy Université, LORIA, Orpailleur Team, Campus Scientifique, BP 239, 54506 Vandœuvre-lès-Nancy Cedex, France

${ }^{2}$ Harmonic Pharma, Espace Transfert, 615 rue du Jardin Botanique, 54600 Villers-lès-Nancy, France

${ }^{3}$ Biosciences eastern and central Africa (BecA) Hub at the International Livestock

${ }^{4}$ Centre International de Référence Chantal Biya (CIRCB) pour la Recherche sur la Prévention et la Prise en charge du VIH/SIDA, BP 3077, Yaoundé, Camerou

\begin{abstract}
The HIV-PDI resource was designed and implemented to address the problems of drug resistance with a central focus on the 3D structure of the target-drug interaction. Clinical and biological data, structural and physico-chemical information and 3D interaction data concerning the targets (HIV protease) and the drugs (ARVs) were meticulously integrated and combined with tools dedicated to study HIV mutations and their consequences on the efficacy of drugs. Here, the capabilities of the HIV-PDI resource are demonstrated for several different scenarios ranging from retrieving information associated with patients to analyzing structural data relating cognate proteins and ligands. HIV-PDI allows such diverse data to be correlated, especially data linking antiretroviral drug (ARV) resistance to a given treatment with changes in three-dimensional interactions between a drug molecule and the mutated protease. Our work is based on the assumption that ARV resistance results from a loss of affinity between the mutated HIV protease and a drug molecule due to subtle changes in the nature of the protein-ligand interaction. Therefore, a set of patients whose resistance to first line treatment was corrected by a second line treatment was selected from the HIV-PDI database for detailed study, and several queries regarding these patients are processed via its graphical user interface. Considering the protease mutations found in the selected set of patients, our retrospective analysis was able to establish in most cases that the first line treatment was not suitable, and it predicted a second line treatment which agreed perfectly with the clincian's prescription. The present study demonstrates the capabilities of HIV-PDI. We anticipate that this decision support tool will help clinicians and researchers find suitable HIV treatments for individual patients. The HIVPDI database is thereby useful as a system of data collection allowing interpretation on the basis of all available information, thus helping in possible decision-makings.
\end{abstract}

\section{Introduction}

The fast growth of HIV resistance to antiretroviral drugs (ARVs) is one of the main limitations in treating the disease [1,2]. Overcoming this resistance in HIV-infected patients is a major public health challenge in AIDS research today [3,4]. The ability of HIV to mutate and produce genetic variations has allowed HIV to develop resistance to many currently available ARVs [5,6]. Crystallographic studies have clearly shown that mutations in the HIV genome can induce structural modifications at the protein active sites targeted by ARVs, which therefore reduce ARV potencies [7-9]. Understanding the mechanisms by which these structural variations emerge and evolve at the molecular level may enable individual patient-specific drug treatments to be formulated. In this context, we developed the HIVPDI (HIV protein drug interaction) resource, which was introduced in the accompanying article (paper 1). The main aim of this resource is to aggregate three-dimensional (3D) structure data relating to HIV-ARV interactions together with more classical biological and clinical data on $\mathrm{HIV}$-infected patients.

While the ultimate goal of the HIV-PDI resource is to help clinical decision-making regarding HIV patients with ARV resistance, it will also facilitate the analysis of HIV resistance for basic research purposes. For example, it allows the progress of patients treated with a first line treatment to be tracked and correlated with structural modifications induced by mutations in the viral proteins. In the first part of this paper, we demonstrate several such analyses which can be performed using HIV-PDI. In the second part of this paper, we validate our basic hypotheses that resistance to treatment stems from a loss of affinity of the delivered ARVs due to mutation-induced structural modifications at the drug binding site, and that similar compensating interaction may be created using other ARVs.

\section{Methods}

\section{HIV-PDI example of use}

HIV-PDI was used as the source of all the data considered in this study to demonstrate the system utilities. HIV-PDI is coupled with tools for visualizing and analyzing 3D Protein-Drug interactions, and with data mining programs. Each query can involve multiple database fields, including the target name, drug name or function, and therapeutic drug classification. This is illustrated here by several requests performed from specific graphic user interfaces (GUIs) in order to extract information and to eventually correlate various data stored in the database. From

*Corresponding authors: GHEMTIO Leo, Nancy Université, LORIA, Orpailleur Team, Campus Scientifique, BP 239, 54506 Vandœuvre-lès-Nancy Cedex, France, Tel: +(33) 3549585 92, Fax: +(33) 3549585 92; E-mail: leo.ghemtio@loria.fr

MAIGRET Bernard, Nancy Université, LORIA, Orpailleur Team, Campus scientifique, BP 239, 54506 Vandoeuvre-lès-Nancy Cedex, France, E-mail: bernard.maigret@loria.fr

OUWE-MISSI-OUKEM-BOYER Odile, Centre International de Référence Chanta Biya (CIRCB) pour la Recherche sur la Prévention et la Prise en charge du VIH/ SIDA, BP 3077, Yaoundé, Cameroun, E-mail: ooukem@gmail.com

Received April 11, 2011; Accepted July 15, 2011; Published July 20, 2011

Citation: GHEMTIO L, SOUCHET M, DJIKENG A, KEMINSE L, KELBERT P, et al. (2011) HIV-PDI: A Protein Drug Interaction Resource for Structural Analyses of HIV Drug Resistance: 2. Examples of Use and Proof-of-Concept. J Health Med Informat 2:105. doi:10.4172/2157-7420.1000105

Copyright: (c) 2011 GHEMTIO L, et al. This is an open-access article distributed under the terms of the Creative Commons Attribution License, which permits unrestricted use, distribution, and reproduction in any medium, provided the original author and source are credited. 
these requests, information or knowledge was extracted by exporting the data into tables and this was a prerequisite of further analysis.

\section{HIV-PDI proof of principle}

From the HIV-PDI database, we selected a subset of patients who presented resistance to a first line ARV treatment, which was overcome by a second line treatment. Given that the HIV-PDI database currently focuses on the well documented HIV protease and related drugs, the only change expected between the two lines of treatment had to be the protease inhibitor. From the biological data recorded in the HIV-PDI database, we retrieved all patients meeting the inclusion criteria by developing a SQL script based queries (PL/pgSQL language). Figure 1 summarizes the three main steps of the selection process. Briefly, for each of the 2029 patients recorded in the HIV-PDI, a chronological list of dates/time was generated, where clinical and/or mutation data were available. Patients who met the following criteria were selected: i) the treatment taken before the first HIV protease sequencing $\left(\mathrm{T}=\mathrm{x}_{1}\right)$ is known, ii) data on viral load, HIV protease sequence and treatment are available at the date of the genotyping test $(\mathrm{T}=0)$, and iii) viral load data are available at the date following the first sequencing $\left(\mathrm{T}=\mathrm{y}_{1}\right)$.
The 3D structures of drug-resistant protease mutants and wild type (WT) protease were selected in an apo form or in complex with protease inhibitors. The process of building the $3 \mathrm{D}$ protease mutants by homology modeling is described in the accompanying article. In order to predict the best molecules that overcome the resistance observed in the selected patients, a docking study was carried out with a collection of 40 NIAID-FDA compounds. The NIAID-FDA collection consists of ARVs from the FDA and NIAID datasets that target the HIV protease. Full details of the docking procedure are described in the accompanying article.

\section{Results}

\section{Cheminformatics analyses}

Searching the HIV-PDI associated database with cheminformatic queries, such as retrieving ligands with a given substructure, searching for or comparing molecules by their 3D shapes, chemical groups, or functions, etc., can help understand the role of chemical moieties with regard to drug resistance. Hence, several cheminformatics routines have been made available in the GUI, e.g. substructure searching and
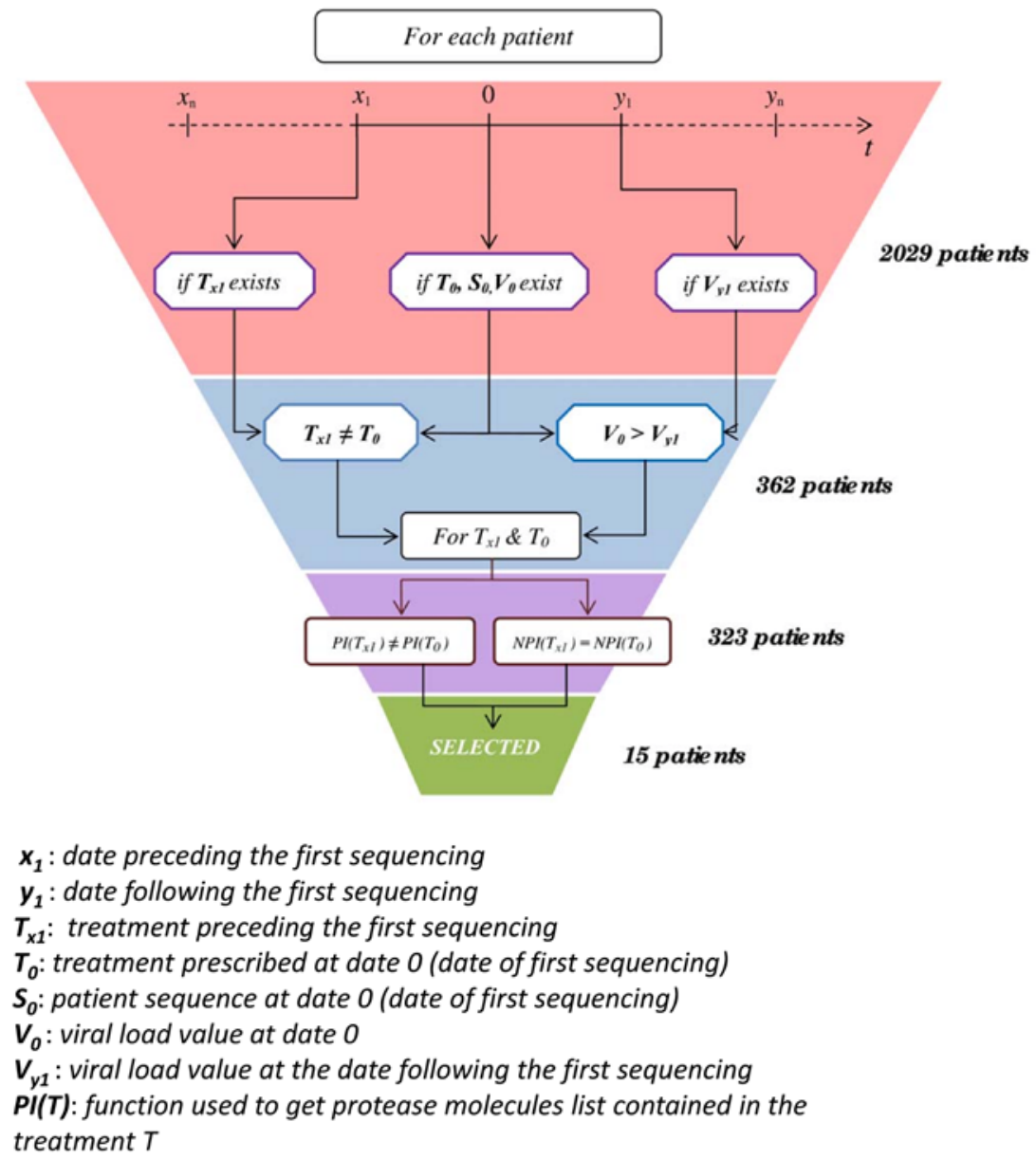

Figure 1: The multistep selection process of the patients from HIV-PDI presenting resistance to an anti-protease which was subsequently overcome with a second anti-protease treatment. 


\begin{tabular}{|c|c|c|c|c|c|c|c|c|c|c|}
\hline & Saquinavir & Ritonavir & Indinavir & Nelfinavir & Amprenavir & Lopinavir & Atazanavir & Fosamprenavir & Tipranavir & Darunavir \\
\hline Saquinavir & 1.00 & 0.54 & 0.59 & 0.57 & 0.50 & 0.57 & 0.61 & 0.52 & 0.45 & 0.50 \\
\hline Ritonavir & 0.54 & 1.00 & 0.51 & 0.44 & 0.52 & 0.59 & 0.55 & 0.55 & 0.52 & 0.55 \\
\hline Indinavir & 0.59 & 0.51 & 1.00 & 0.53 & 0.45 & 0.52 & 0.54 & 0.46 & 0.42 & 0.45 \\
\hline Nelfinavir & 0.57 & 0.44 & 0.53 & 1.00 & 0.45 & 0.52 & 0.47 & 0.46 & 0.40 & 0.46 \\
\hline Amprenavir & 0.50 & 0.52 & 0.45 & 0.45 & 1.00 & 0.48 & 0.51 & 0.87 & 0.49 & 0.89 \\
\hline Lopinavir & 0.57 & 0.59 & 0.52 & 0.52 & 0.48 & 1.00 & 0.55 & 0.48 & 0.41 & 0.49 \\
\hline Atazanavir & 0.61 & 0.55 & 0.54 & 0.47 & 0.51 & 0.55 & 1.00 & 0.53 & 0.47 & 0.53 \\
\hline Fosamprenavir & 0.52 & 0.55 & 0.46 & 0.46 & 0.87 & 0.48 & 0.53 & 1.00 & 0.53 & 0.81 \\
\hline Tipranavir & 0.45 & 0.52 & 0.42 & 0.40 & 0.49 & 0.41 & 0.47 & 0.53 & 1.00 & 0.54 \\
\hline Darunavir & 0.50 & 0.55 & 0.45 & 0.46 & 0.89 & 0.49 & 0.53 & 0.81 & 0.54 & 1.00 \\
\hline
\end{tabular}

Table 1: Tanimoto similarity scores between usual ARVs extracted from HIV-PDI. High scores represent high similarities. Here compounds highly similar to amprenavir are highlighted in grey.

Tanimoto similarity calculations. For this purpose, the main GUI page (see paper 1 for its description), contains search possibilities on ligand categories in order to retrieve the file containing the required properties to be used for further analyses in the database. For example it is possible to specify the $2 \mathrm{D}$ or $3 \mathrm{D}$ chemical structure of any ligand as well as its InChIKey [10], or Chemaxon fingerprints [11]. This allows all similar compounds in the database to retrieved, or all compounds that were selected by chemical groups or molecular structure to be identified (see Table 1, which shows the tanimoto similarity scores between usual ARVs extracted from HIV-PDI). More advanced analyses can also be carried out, such as retrieving ligands with a similar shape, chemical substituents or pharmacophores to those of a given query ligand.

\section{General structural analysis}

Structural data relating to mutants and WT Protein Data Bank (PDB) structures of the HIV-1 protease are stored in the HIV-PDI database. This information can also be retrieved through the GUI. It is therefore possible to investigate the influence of given mutations on the 3D structures of the target proteins, to compare their 3D structures and to highlight their differences. Importantly, modifications of ARVprotease interaction patterns in mutant proteases can be investigated at the 3D structure level using the GUI in combination with the Jmol, an open-source Java viewer for three-dimensional chemical structures [12]. For example, the main structural characteristics of the protease homo-dimer can easily be visualized for each structure in the database. Figure 2a shows the Asp25 position of the critical Asp25-Thr26-Gly27 triad of the active site $[13,14]$, in the vicinity of which subsequent studies could be focused on. Other structural elements such as the anti-parallel $\beta$-sheets linking the two subunits and the so-called "flap structure" [15] in each subunit can also be inspected manually.

The structural differences that exist between the bound and free states of the proteins can also be visualized and compared (Figure 2b) $[13,14]$. Such analyses reveal that, in all of the holo structures, the flaps are pulled inward toward the bottom of the active site (the "closed" form), whereas a "semi open" conformation is observed in the structures of the apo protease, with the flaps shifted away from the dual catalytic aspartate (Asp25) but still forming a lid over the active site and still maintaining contacts with each other. Such an analysis can be extended, for example, to compare the $3 \mathrm{D}$ interaction patterns of the holo structures containing a given class of ligand, or to compare all WT protein structures complexed with different ligands.
Many other types of queries are supported by the HIV-PDI, such as getting a list of all existing WT structures (see Table 2, which shows the currently available WT structures of HIV-1 protease in the PDB), of requesting all existing mutated structures which are linked to resistance of a particular ARV (see Table 3, which shows the HIV-1 mutations that confer resistance to the main protease inhibitors). All such lists can be analyzed and the associated 3D structures compared and dissected at both the protease and the ligands levels.

The HIV-PDI system can also be used to list the different types of mutations found in the protease (Table 3), and to consider their influence according to their $3 \mathrm{D}$ location within the protein structure [16-21]. For example, all of the available protease structures to date (see Table 4, which shows the protease structures complexed with amprenavir) in complex with the drug amprenavir (APV) were extracted from the HIV-PDI database and compared. Calculating the root mean squared deviation (RMSD) deviations between the $\mathrm{Ca}$ backbone atoms in these complexes shows that the overall protease conformation is maintained with an RMSD of $0.7 \AA$, regardless of the locations of the mutations (Figure 2c). Nonetheless, some small local flap modifications between mutations are observed, and these could be associated with changes in ARV binding affinities in the active site, thus explaining reduced activity of the protease inhibitors in those mutants $[14,22,23]$.

Another way to investigate ARV resistance is to analyze modifications of favorable drug binding interactions with the mutated proteins that appear during HIV infection [24,25]. Several recent papers have highlighted the importance of considering such interaction data to understand resistance [26-42]. Our HIV-PDI system makes it possible to do this at a molecular level. For example, Figure $2 \mathrm{~d}$ shows a comparison between the crystal structures of the WT HIV protease (PDB code 3EKV) and the I50L/A71V mutant (PDB code 3EM3), both in complex with APV, which illustrates this kind of analysis.

\section{Patient-specific HIV protease mutation analyses}

HIV-PDI can also be used to extract patient data and to compare the associated structural information to those found in the PDB. This is demonstrated here using patient 39546, who was selected at random selected from the HIV-PDI database, and whose HIV protease was found to have the following mutations: L15V, E35D, R41K, I50L, and 

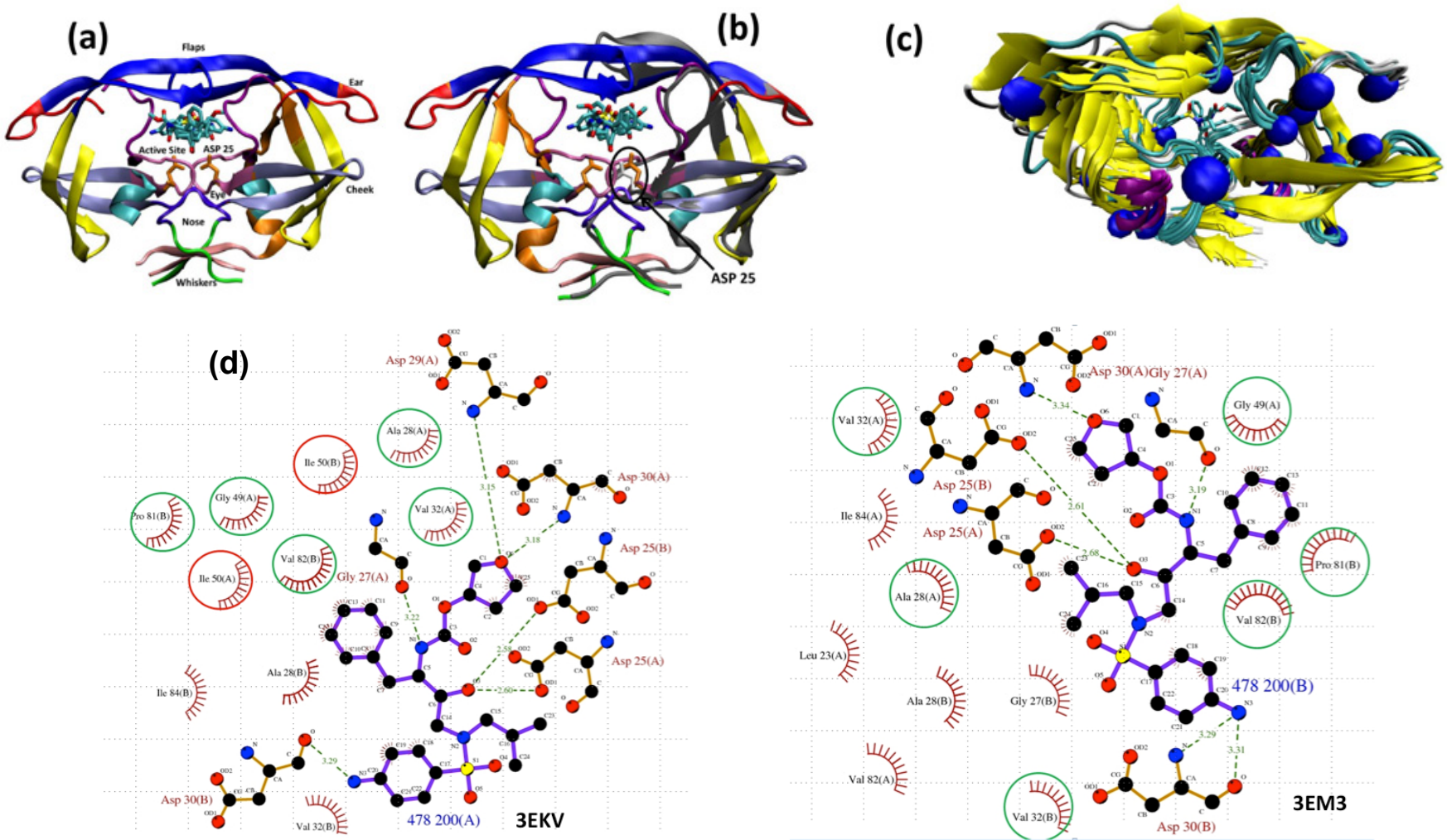

Figure 2: Overall topology and molecular interactions of PDB HIV protease in apo and halo complexes. (a): The topology [15] of HIV-1 protease crystal structure of 2 qmp in complex with two A00 molecules (sticks).

The usual terminology of the topology of HIV protease includes the following: flap (43-58), ear (35-42), cheek (cheek turn 11-22 and cheek sheet 59-75), eye (23-30), whisker (1-5 and 95-99), and nose (6-10).

(b): Superpositions of the wild-type apo protease (PDB code 3PHV; for clarity, only one monomer unit is displayed as a grey ribbon on the right hand side), and the homodimer of the wild-type holo protease complex with two A00 molecules (PDB code 2AMP; ribbon colored according to secondary structures).

The critical Asp25 of the apo form displayed in white sticks moved downward with regards to the Asp25 of the complex in orange sticks. The flap of the apo form moved upward compare to the flap of complex protease.

(c): Ca superposition of all 12 PDB structures complexed with amprenavir (see Table, Supplemental Digital Content 4, which shows the protease structures complexed with amprenavir). The mutation locations are shown as blue spheres.

(d): Ligplot graph showing the change in the protease/amprenavir interactions between the WT protease (PDB code 3EKV) and the drug resistant HIV-1 protease variant (I50L/A71V; PDB code 3EM3): The green circles represent conserved interactions, the red circles represents the mutated residues lost in $3 E M 3$.

V82L. Extracting the clinical data for this patient shows that ARV resistance in this patient was revealed by virological failure (increased viremia) after first line treatment with the amprenavir (APV) antiprotease. By comparing the protein-ligand complex of this patient's protease with a drug resistant HIV-1 mutant from the PDB both with the crystal structure of WT HIV-1 protease, the presence of several structural differences between the two structures can be observed (Figure 3a). The flaps of the PDB structure and the patient mutant are more open than the flap of the WT structure. Furthermore, the ear, whiskers, nose, eye and the two active sites are also slightly modified. Even though the protease in patient 39546 has more mutations (Figure 3a, panel A) than the EM3 drug resistant mutated protease from the PDB (Figure 3a, panel B), the majority of this patient's mutations are far from the protease active site, except for I50L which is situated on the flap very close to the active site. This suggests that the main mutation that influences ARV the activity is the same (i.e. I50L) in these two proteases.
Another type of evidence that may be obtained from HIV-PDI concerning this patient is the shape of the binding site cavity in the mutated protease. The effect of mutations on the structural features of the binding cavity may be analysed in order to explain variations in potency observed for a given drug and a mutated HIV-1 protease. For example, the WT HIV-1 protease structure (PDB code 3EKV), the structure of the $150 \mathrm{~L} / \mathrm{A} 71 \mathrm{~V}$ protease mutant (PDB code 3EM3), and a homology-modeleted structure of the protease structure of patient 39546 may be compared by superposing their respective structures. Figure $3 \mathrm{~b}$ shows the shapes of the three cavities calculated using our spherical harmonic comparison technique developed previously by us. This Figure shows that although the three cavities have broadly similar shapes, the cavity of the patient is considerably smaller than those of the other two structures. This difference could explain why the two mutated HIV-1 proteases do not have the same affinity for APV comparised to the WT HIV-1. This will be confirmed by an analysis of the pattern of intermolecular interactions between the WT and mutated HIV-1 protease complexed with APV. 

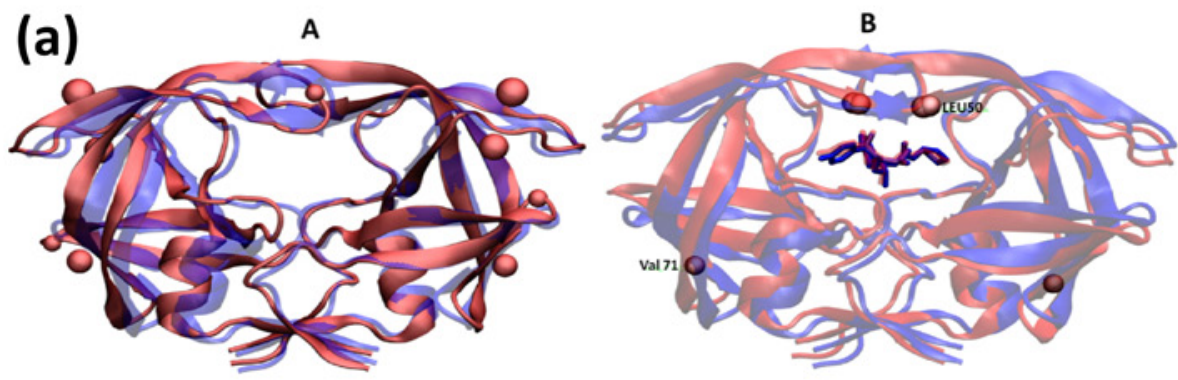

(b)
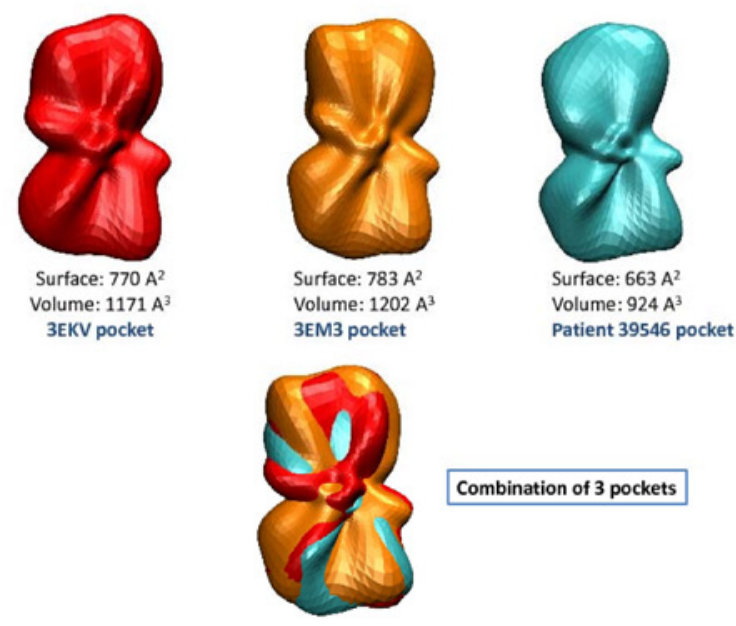

Combination of 3 pockets

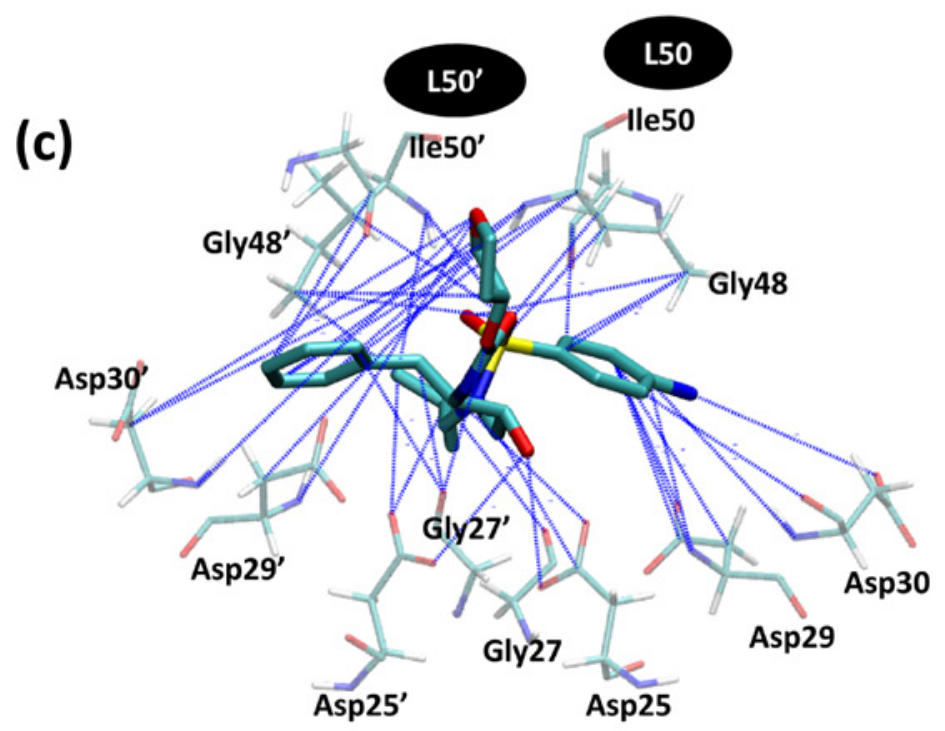

Figure 3: Several comparisons of mutated HIV protease with the WT protease.

(a): The effect of mutations on 3D structures of the HIV-1 protease. The location of each mutation is shown as a ball.

Panel A: superposition of the HIV-1 protease 3D structure of patient 39546 (with L15V, E35D, R41K, 150L, V82 mutations) displayed in red (sequencing at time T=0 in HIV-PDI) with the crystal structure of the WT HIV-1 protease shown in purple (PDB entry: 3EKV). Panel B: superposition of the I50L/A71V drug-resistant HIV-1 protease mutant (in red; PDB entry: 3EM3) with the crystal structure of the WT HIV-1 protease (in blue; PDB entry: 3EKV). The two proteases are complexed with APV (displayed as sticks).

(b): Spherical harmonic based representations of ligand binding cavities.

The cavities of WT HIV-1 protease (PDB code 3EKV), I50L/A71V Drug-Resistant HIV-1 Protease Mutant (PDB code 3EM3), and the HIV-1 protease model structure of patient 39546 are displayed in red, orange, and light blue, respectively.

(c): Visualization of the APV interaction pattern

The main protease residues (Asp25, Gly27, Asp29, Asp30, Gly48, lle50) [24, 25] necessary for drug binding are displayed as sticks. Blue lines highlight interactions lost compared to the WT protease (PDB entry 3EKV) when APV is docked to the $150 \mathrm{~L}$ mutated protease of patient 39546. 


\begin{tabular}{|l|l|l|l|}
\hline PDB ID & Resolution $(\mathbf{A})$ & Strain & Ligand ID \\
\hline 1 A8G & 2.50 & WT & HV8 \\
\hline 1 EBY & 2.29 & WT & BEB \\
\hline 1 HXW & 1.80 & WT & RIT \\
\hline 1 IZH & 1.90 & WT & Q50 \\
\hline 1 PRO & 1.80 & WT & A88 \\
\hline 1 SBG & 2.30 & WT & IM1 \\
\hline 1 VIJ & 2.40 & WT & BAY \\
\hline 1 VIK & 2.40 & WT & BAY \\
\hline $2 \mathrm{HVP}$ & 3.00 & WT & N/A \\
\hline 2 QMP & 1.80 & WT & A00 \\
\hline 3 M9F & 1.80 & WT & 595 \\
\hline 4 PHV & 2.10 & WT & VAC \\
\hline $5 H V P$ & 2.00 & WT & STA \\
\hline $9 H V P$ & 2.80 & WT & COR \\
\hline
\end{tabular}

Table 2: The currently available WT structures of HIV-1 protease in the PDB.

\begin{tabular}{|l|l|l|}
\hline & \multicolumn{2}{|l|}{ Position(s) in protease } \\
\hline Protease inhibitors & Critical substitutions & Additional substitutions \\
\hline Saquinavir & 48,90 & $10,36,63,71$ \\
\hline Ritonavir & 82,84 & $20,36,46,54,63,71,90$ \\
\hline Indinavir & 46,82 & $10,20,24,32,54,63,71,84,90$ \\
\hline Nelfinavir & 30 & $46,63,71,88,90$ \\
\hline Amprenavir & 50 & $10,46,47$ \\
\hline
\end{tabular}

Table 3: The HIV-1 mutations that confer resistance to the main protease inhibitors. [16-21].

\begin{tabular}{|l|l|}
\hline PDB codes & Observed mutations \\
\hline 1HPV & N37S \\
\hline 1T7J & Q7K,K14R,L63P,V82T,I84V \\
\hline 3EKP & Q7K,L10I,K14R,R41K,G48V,I54V,L63P,V82A \\
\hline 3EKV & Q7K,K14R,R41K,L63P \\
\hline 3EM3 & Q7K,K14R,R41K,I50L,L63P,A71V \\
\hline 3NU3 & Q7K,L33I,N37S,L63I,C67A,C95A \\
\hline 3NU4 & Q7K, V32I,L33I,N37S,L63I,C67A,C95A \\
\hline 3NU5 & Q7K,L33I,N37S,I50V,L63I,C67A,C95A \\
\hline 3NU6 & Q7K,L33I,N37S,I54M,L63I,C67A,C95A \\
\hline 3NU9 & Q7K,L33I,N37S,L63I,C67A,I84V,C95A \\
\hline 3NUJ & Q7K,L33I,N37S,I54V,L63I,C67A,C95A \\
\hline 3NUO & Q7K,L33I,N37S,L63I,C67A,L90M,C95A \\
\hline
\end{tabular}

Table 4: The protease structures complexed with Amprenavir.

Intermolecular interactions concerning one or several proteinligand complexes can also be obtained from the HIV-PDI database, and these are helpful to explain drug affinity changes due to mutations in the protease. For example, as shown in Figure 3c, several interactions have been lost in the mutated protease complex of patient 39546.
These interactions concern residues of both the flap and the active site regions, which are necessary for inhibition of the protease [24,25].

\section{Structural differences between patients' mutants and WT apo protease}

According to our working hypothesis regarding the link between drug resistance and modifications of protein-drug interaction patterns, we selected a subset of patients whose resistance to first line treatment was overcome by a second therapy. A set of 362 patients matched the first criteria of a multi step selection process, which finally gave a group of 15 patients (Figure 1). While this number is very small, it nonetheless provides a genuine test of the validity of our hypothesis. Table 5 lists detailed data related to the selected set of patients. The mutations present on the patients' protease concern several locations where important residues for drug binding have been identified (see Table 6, which shows the residue mutations observed in the 15 patients) [16-21].

The overall model structures of the HIV-1 apo protease mutants isolated from the 15 patients are similar to the WT protease structure with a RMSD of between 0.9-1.5 A for all main chain Ca atoms. The mutations present in each patient's protease caused weak modifications of the global geometry of the protein (see Figure, Supplementary information 1, which shows the Superposition of all 15 patients mutated apo proteases and the 2QMP WT structure). In fact, when looking in more detail, the largest differences between the WT and the patient's mutant structures are seen in the most flexible protein regions, namely the flaps, ear, and cheek, while the catalytic triads of residues 25-27 and $25^{\prime}-27^{\prime}$ show very low main chain atom deviations. These changes are similar to the structural changes observed experimentally in the whole set of PDB mutants, as may be confirmed by checking all the PDB files stored in the HIV-PDI resource.

\section{Analysis of the binding mode of the antiprotease drugs in the patient mutated proteases}

The 3D protein-ligand structures for the 15 patients' proteases and the 3 ARVs involved in these patients' first and second line treatments (namely indinavir, saquinavir, nelfinavir) were extracted from the HIVPDI database and compared. These holo structures were obtained by selecting the best poses of the ligands deriving from molecular docking. For all the 15 selected patients, indinavir had a better docking score than the other tested molecules and especially saquinavir (see Table 7, which shows the patient-related data and best compound docking solutions). Of course such a result is only a preliminary indication since a deeper analysis of the protein-ligand interactions is necessary to rank the docked compounds.

At this stage, it is important to compare the detailed molecular interactions found in the selected 15 patients' protease mutants (see Table 8, which shows the critical residues involved in ARV interactions and characterized in the crystal HIV-1 proteases from HIV-PDI database) with the $3 \mathrm{ARV}$ complexes considered above to those present in PDB complexes (see Table 9, which shows the list of PDB entry code related to HIV-1 protease) with the same ligands. In order to illustrate use of these data, the $3 \mathrm{D}$ structure of the drug-resistant protease mutant of one selected patient (patient 6670) and the WT structure protease (PDB code 2QMP) were compared in complex with both saquinavir and indinavir, respectively. This example is typical of all 15 patients except the 19255 one. The comparison of the lists of residues involved in key interactions with the ligands shows that 
Citation: GHEMTIO L, SOUCHET M, DJIKENG A, KEMINSE L, KELBERT P, et al. (2011) HIV-PDI: A Protein Drug Interaction Resource for Structural Analyses of HIV Drug Resistance: 2. Examples of Use and Proof-of-Concept. J Health Med Informat 2:105. doi:10.4172/2157-7420.1000105

Page 7 of 1

\begin{tabular}{|c|c|c|c|c|c|c|c|c|c|c|}
\hline Patient ID & $t_{x}^{*}$ & $\mathbf{V}_{\mathrm{x}}{ }^{*}$ & $T_{x}{ }^{*}$ & $\mathbf{t}_{0}{ }^{*}$ & $V_{0}{ }^{*}$ & $\mathrm{CD} 4{ }_{0}{ }^{*}$ & $\mathrm{~T}_{0}{ }^{*}$ & $t_{y}{ }^{*}$ & $\mathbf{V}_{\mathrm{y}}{ }^{*}$ & $\mathrm{CD} 4_{y}{ }^{*}$ \\
\hline 6652 & -1 & 3.9 & AZT DDC SQV & 0 & 3.8 & 179 & AZT DDC IDV & 2 & 3.6 & 132 \\
\hline 6665 & -2 & 4.4 & 3TC AZT SQV & 0 & 4.2 & 93 & 3TC AZT IDV & 2 & 3.9 & 90 \\
\hline 6670 & -2 & 5 & 3TC D4T SQV & 0 & 5 & 22 & 3TC D4T IDV & 2 & 3.3 & 40 \\
\hline 6671 & -2 & 4.3 & DDC SQV & 0 & 4.7 & 401 & DDC IDV & 2 & 4.4 & 330 \\
\hline 6674 & -2 & 4.9 & 3TC AZT SQV & 0 & 4.6 & 286 & 3TC AZT IDV & 2 & 2.6 & 322 \\
\hline 6678 & -2 & 3.6 & 3TC AZT SQV & 0 & 3.9 & 639 & 3TC AZT IDV & 2 & 2.8 & 835 \\
\hline 6685 & -1 & 4.8 & 3TC AZT SQV & 0 & 4.8 & 165 & 3TC AZT IDV & 2 & 4 & 178 \\
\hline 6686 & -1 & 4.8 & 3TC AZT SQV & 0 & 4.8 & 55 & 3TC AZT IDV & 2 & 3.1 & 114 \\
\hline 6687 & -1 & 3.7 & 3TC AZT SQV & 0 & 3.7 & 188 & 3TC AZT IDV & 1 & 3 & 184 \\
\hline 6689 & -4 & 2.5 & AZT DDC SQV & 0 & 2.8 & 254 & AZT DDC IDV & 2 & 2.2 & 250 \\
\hline 6691 & -1 & 4.9 & 3TC AZT SQV & 0 & 4.8 & 82 & 3TC AZT IDV & 2 & 2.5 & 92 \\
\hline 6693 & -3 & 3.1 & 3TC D4T SQV & 0 & 4 & 627 & 3TC D4T IDV & 2 & 2.7 & 459 \\
\hline 6696 & -1 & 3.5 & 3TC AZT SQV & 0 & 3.7 & 157 & 3TC AZT IDV & 2 & 2.3 & 180 \\
\hline 6706 & -1 & 4.3 & 3TC AZT SQV & 0 & 4.3 & 284 & 3TC AZT IDV & 1 & 3.1 & 233 \\
\hline 19255 & -140 & & 3TC D4T IDV & 0 & 5.2 & 18 & 3TC D4T NFV & 13 & 3.1 & 91 \\
\hline
\end{tabular}

${ }^{*} \mathrm{~T}=$ Treatment, $\mathrm{t}=$ time (week), $\mathrm{V}=\mathrm{viremia}$; CD4

Table 5: The biological data of the 15 selected patients. ARV resistance was observed at time t0, and a rescue in inhibitory activity was measured at time ty.

\begin{tabular}{|c|c|c|c|}
\hline Patient ID & Mutations & Treatment1 & Treatment2 \\
\hline 6652 & K14V-I15V-D60E & SQV & IDV \\
\hline 6665 & L10I-K14R-L63P-A71V-G73S-V77I-L90M-I93L & SQV & IDV \\
\hline 6670 & L10I-G16E-P39T-G48V-I54V-D60E-L63P-A71T-V77I-L90M & SQV & IDV \\
\hline 6671 & L10I-I62V-L63P-G73S-V77I-L90M & SQV & IDV \\
\hline 6674 & N37D-Q58E-L63S-A71T-172T-V77I & SQV & IDV \\
\hline 6678 & K43R-L63P-A71T-I93L & SQV & IDV \\
\hline 6685 & L10I-K14R-L19I-E35G-Gㅆ8V-F53L-L63A-A71V-I72V-T74S-V77I-V82A & SQV & IDV \\
\hline 6686 & 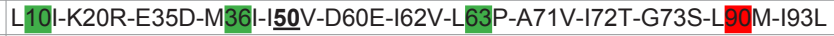 & SQV & IDV \\
\hline 6687 & I13V-I15V-L63A-172M & SQV & IDV \\
\hline 6689 & I15V-E35D-M36I-N37A-I62V-L63P-G73S-I85V-L90M & SQV & IDV \\
\hline 6691 & E35D-N37D-L63P-A71|-L90M-I93L & SQV & IDV \\
\hline 6693 & T12S-I13V-K14R-G16E-E35D-N37A-L63Q-I64V & SQV & IDV \\
\hline 6696 & 115V-P39T-A71T-L89P-L90M & SQV & IDV \\
\hline 6706 & N37S-L63R-K70V-V77I-I93V & SQV & IDV \\
\hline 19255 & M36I-L63P & IDV & NFV \\
\hline
\end{tabular}

*Saquinavir (SQV) critical substitutions: red color

SQV additional substitutions: green color

**Indinavir (IDV) critical substitutions: red color

IDV additional substitutions: yellow color

Important residues for drug binding and stability: Asp25, Gly 27, Asp29, Asp30, Gly48, lle50

Table 6: Residue mutations observed in the 15 patients colored according to their known effect on Saquinavir* and Indinavir* ${ }^{\star *}$ resistance. The analysis is focused on the catalytic residues important residues for drug binding and stability (Asp25, Gly 27, Asp29, Asp30, Gly48, Ile50), the residues of the active site (Asp25-Thr26-Gly27), and the residues provoking specific substitutions (see Table, Supplemental Digital Content 3, which shows the HIV-1 mutations that confer resistance to the main protease inhibitors).

\begin{tabular}{|l|l|l|l|l|l|l|l|l|l|}
\hline Patient ID & Treatment 1 & \multicolumn{9}{c|}{ Treatment 2 } & & \multicolumn{2}{l|}{ Best pose } \\
\hline & Treatment & Protease & Rank & Score & Treatment & Protease & Rank & Score & Name \\
\hline 6652 & AZT DDC SQV & SQV & 3 & -8.9 & AZT DDC IDV & IDV & 1 & -11.0 & IDV \\
\hline 6665 & 3TC AZT SQV & SQV & 6 & -7.0 & 3TC AZT IDV & IDV & 1 & -9.5 & IDV \\
\hline 6670 & 3TC D4T SQV & SQV & 10 & -7.1 & 3TC D4T IDV & IDV & 1 & -9.1 & IDV \\
\hline 6671 & DDC SQV & SQV & 2 & -8.7 & DDC IDV & IDV & 1 & -9.2 & IDV \\
\hline 6674 & 3TC AZT SQV & SQV & 2 & -9.3 & 3TC AZT IDV & IDV & 1 & -9.9 & IDV \\
\hline 6678 & 3TC AZT SQV & SQV & 3 & -7.9 & 3TC AZT IDV & IDV & 1 & -9.9 & IDV \\
\hline 6685 & 3TC AZT SQV & SQV & 4 & -7.3 & 3TC AZT IDV & IDV & 1 & -10.7 & IDV \\
\hline 6686 & 3TC AZT SQV & SQV & 3 & -9.3 & 3TC AZT IDV & IDV & 1 & -10.0 & IDV \\
\hline 6687 & 3TC AZT SQV & SQV & 3 & -8.8 & 3TC AZT IDV & IDV & 1 & -10.7 & IDV \\
\hline 6689 & AZT DDC SQV & SQV & 2 & -9.5 & AZT DDC IDV & IDV & 1 & -10.5 & IDV \\
\hline 6691 & 3TC AZT SQV & SQV & 3 & -8.7 & 3TC AZT IDV & IDV & 1 & -9.3 & IDV \\
\hline 6693 & 3TC D4T SQV & SQV & 6 & -7.4 & 3TC D4T IDV & IDV & 1 & -10.8 & IDV \\
\hline 6696 & 3TC AZT SQV & SQV & 2 & -8.3 & 3TC AZT IDV & IDV & 1 & -10.1 & IDV \\
\hline 6706 & 3TC AZT SQV & SQV & 3 & -8.8 & 3TC AZT IDV & IDV & 1 & -10.6 & IDV \\
\hline 19255 & 3TC D4T IDV & IDV & 1 & -10.7 & 3TC D4T NFV & NFV & 8 & -7.1 & IDV \\
\hline
\end{tabular}

AZT (Zidovudin), DDC (Zalcitabin), SQV (Saquinavir), IDV (Indinavir), 3TC (Lamivudin), D4T (Stavudin), NFV (Nelfinavir).

Table 7: Patient-related data and best compound docking solutions. Patient-related data and best compound docking solutions according to Glide score (lower scores are best). 
Citation: GHEMTIO L, SOUCHET M, DJIKENG A, KEMINSE L, KELBERT P, et al. (2011) HIV-PDI: A Protein Drug Interaction Resource for Structural Analyses of HIV Drug Resistance: 2. Examples of Use and Proof-of-Concept. J Health Med Informat 2:105. doi:10.4172/2157-7420.1000105

Page 8 of 11

\begin{tabular}{|c|c|c|c|c|c|}
\hline \multicolumn{2}{|c|}{$\begin{array}{l}\text { Interactions present in all } 14 \text { Indinavir PDB } \\
\text { complex structures presently available in HIV- } \\
\text { PDI }\end{array}$} & \multicolumn{2}{|c|}{$\begin{array}{l}\text { Interactions present in all } 10 \text { Nelfinavir PDB } \\
\text { complex structures presently available in HIV-PDI }\end{array}$} & \multicolumn{2}{|c|}{$\begin{array}{l}\text { Interactions present in all } 14 \text { Saquinavir PDB } \\
\text { complex structures presently available in HIV-PDI }\end{array}$} \\
\hline Residue & Number & Residue & Number & Residue & Number \\
\hline ALA & $28 \mathrm{~A}$ & ALA & $28 \mathrm{~A}$ & ALA & $28 \mathrm{~A}$ \\
\hline ALA & 28B & ALA & $28 \mathrm{~B}$ & ASP & $25 \mathrm{~A}$ \\
\hline ARG & $\begin{array}{l}8 \mathrm{~A} \\
25 \mathrm{~A}\end{array}$ & ARG & 8B & ASP & $29 A$ \\
\hline ASP & \begin{tabular}{|l|}
$25 \mathrm{~A}$ \\
$25 \mathrm{~B}$ \\
\end{tabular} & ASP & $25 \mathrm{~A}$ & ASP & $30 \mathrm{~A}$ \\
\hline ASP & $\begin{array}{l}25 B \\
29 A\end{array}$ & ASP & $25 B$ & GLY & $27 \mathrm{~A}$ \\
\hline NCD & 500 & ASP & $29 \mathrm{~A}$ & GLY & $49 A$ \\
\hline ASP & 200 & GLY & $27 \mathrm{~A}$ & ILE & $47 \mathrm{~A}$ \\
\hline ASP & 30A & GLY & 27B & LEU & $23 \mathrm{~A}$ \\
\hline ASP & 3UB & GLY & $49 B$ & PRO & $81 \mathrm{~A}$ \\
\hline GLY & $27 \mathrm{~A}$ & \multirow{2}{*}{ ILE } & $47 \mathrm{~A}$ & & \\
\hline GLY & 27B & & & & \\
\hline GLY & $48 \mathrm{~A}$ & ILE & 47B & & \\
\hline GLY & $48 \mathrm{~B}$ & ILE & $50 \mathrm{~A}$ & & \\
\hline GLY & $49 A$ & ILE & $50 \mathrm{~B}$ & & \\
\hline GLY & $49 \mathrm{~B}$ & LEU & $23 \mathrm{~A}$ & & \\
\hline GLT & 490 & LEU & 23B & & \\
\hline LEU & $23 \mathrm{~A}$ & PRO & $81 \mathrm{~A}$ & & \\
\hline LEU & 23B & PRO & 81B & & \\
\hline PRO & $81 \mathrm{~A}$ & VAL & $32 \mathrm{~A}$ & & \\
\hline PRO & 81B & VAL & $32 \mathrm{~B}$ & & \\
\hline
\end{tabular}

Table 8: Critical residues involved in ARV interactions and characterized in the crystal HIV-1 proteases from HIV-PDI database. Critical residues involved in ARV interactions and characterized in the crystal HIV-1 proteases from HIV-PDI database in complex with Indinavir, Nelfinavir, and Saquinavir, respectively.

\begin{tabular}{|c|c|c|c|c|c|c|c|c|c|c|c|c|c|c|}
\hline \multirow{2}{*}{\begin{tabular}{|l} 
Compound \\
indinavir \\
\end{tabular}} & \multicolumn{14}{|c|}{ PDB Complex } \\
\hline & $1 \mathrm{C} 6 \mathrm{Y}$ & 1HSG & $1 \mathrm{HSH}$ & $1 \mathrm{~K} 6 \mathrm{C}$ & 1SDT & 1SDU & 1SDV & 1SGU & $2 \mathrm{AVO}$ & 2AVS & $2 A V V$ & 2B7Z & 2BPX & 2R5P \\
\hline nelfinavir & 1OHR & 2PYM & 2PYN & 2Q63 & 2Q64 & 2QAK & $2 \mathrm{R} 5 \mathrm{Q}$ & $3 E K X$ & 3EL0 & 3EL5 & & & & \\
\hline saquinavir & $1 \mathrm{C} 6 \mathrm{Z}$ & 1FB7 & 2NMW & $2 \mathrm{NMY}$ & $2 \mathrm{NMZ}$ & $2 N N K$ & 2NNP & $3 C Y X$ & 3D1X & 3D1Y & 3EKQ & 3EL4 & $3 \mathrm{~K} 4 \mathrm{~V}$ & $3 N 3 I$ \\
\hline
\end{tabular}

Table 9: List of PDB entry code related to HIV-1 protease. List of PDB entry code related to HIV-1 protease structures in complex with Indinavir, Nelfinavir and Saquinavir, respectively.

several of them no longer form the canonical interactions (see Figure), Supplementary information 2, which shows the counts of critical protease-ARV interactions for saquinavir and indinavir in the WT and patient 6670 proteases). Indeed, the data (Supplementary information 2) show that on balance, the interactions between saquinavir in the wild type (the expected interactions) and in the mutant protease of patient 6670 are less favorable compared to those of indinavir, and that the total numbers of interactions corresponds exactly the docking ranking. In the case of patient 19255, if we consider only the docking rank of nelfinavir which was used as the second line treatment, this compound seems less favorable compared to indinavir which was used as the first line treatment (rank 8 versus first rank, respectively). If we now consider the interaction network based re-scoring, the differences in the ARV-protease interactions obtained for both indinavir-19255 protease and nelvinavir-19255 protease, especially focused on the socalled critical residues, it appears that the nelfinavir complex presents $36 \%$ more critical interactions compared to the indinavir complex (see Table 10). It would therefore be possible to propose nelfinavir as the best second line compound when compared to indinavir. This proposal corresponds to the treatment effectively given to the patient 19255.

\section{Discussion}

This article has highlighted some of the novel uses of the HIVPDI resource. Compared to other databases focusing on HIV resistance, the innovative possibilities offered in HIV-PDI are in good accordance with recent trends in dealing with HIV drug resistance. For example, using chemoinformatics tools can contribute towards making better decisions in HIV drug discovery processes [43-47]. The chemoinformatics capabilities available in HIV-PDI are demonstrated. As presented above, when faced with resistance to a given compound such as APV, the Tanimoto score may be used to suggest possible rescue treatments. For example, according to their Tanimoto scores, darunavir, fosamprenavir, and amprenavir" should be replaced by "Darunavir, Fosamprenavir and Amprenavir and consequently should be eliminated because they might have the same unfavourable behaviour towards their mutated protein target. On the other hand, if for some reason a particular anti-protease drug is not available, one can choose the next closest one according to its Tanimoto score. 


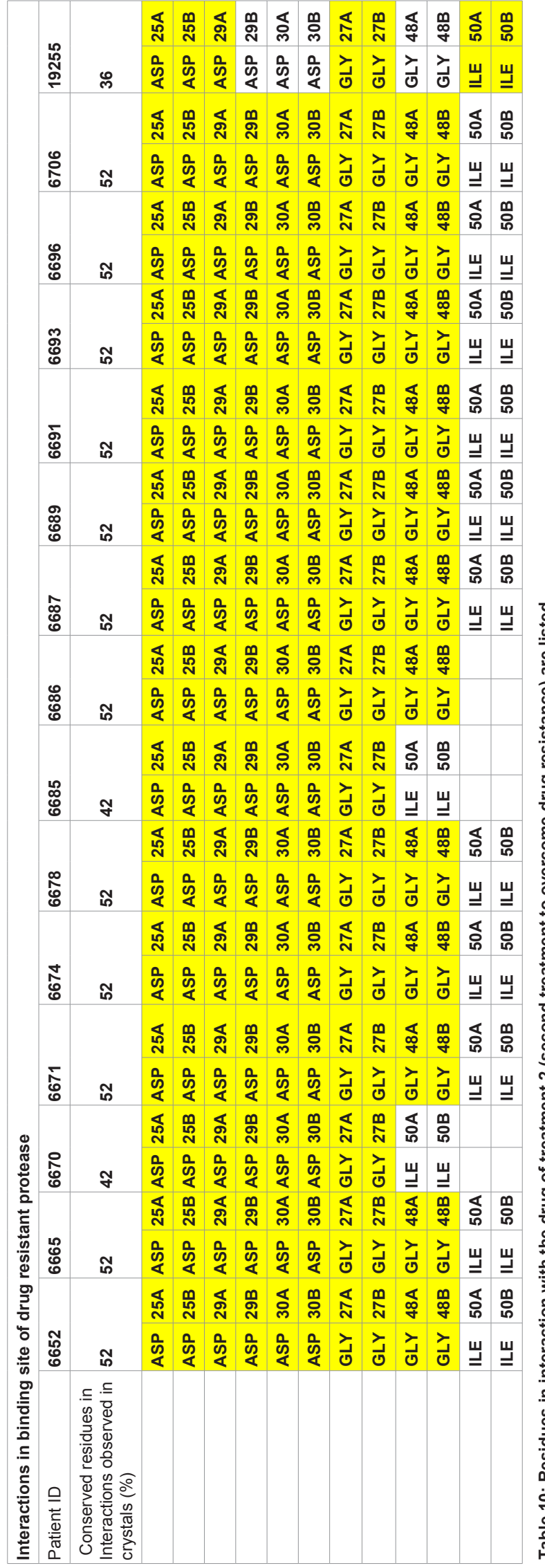

Similarly, the interest for performing binding site cavity analyses like those presented here is also very topical as an important current approach $[48,49]$. From such analyses, as demonstrated here for patient 39546, the binding capabilities of APV can be predicted to be modified his mutated protease due to the reduced size of the binding pocket, in agreement with the preceding analyses on $3 \mathrm{D}$ pattern interactions. Therefore, future work will include clustering of binding cavity or ligand using the shape descriptors together with target information and activity classes from HIV-PDI database to predict putative targets for new small molecule structures [50].

One novel capability of HIV-PDI is to provide 3D structures for all the protease mutants. While most of these structures are built by homology, in the case of the HIV protease it has been reported that such models are reliable enough to be used or molecular docking studies [51]. The capability of HIV-PDI for refining these homology models through MD simulations in explicit solvent would provide users with good quality $3 \mathrm{D}$ mutated protease structures. MD has proved to be a suitable modeling technique for HIV proteases [52].

Numerous docking studies on HIV resistance have been reported [32]. In this context, several publications have highlighted some of the limitations on the ability of available docking programs to predict correctly ligand binding affinities [53]. This is why the HIVPDI resource uses additional criteria such as ligand comparisons (Table 1) and counting of critical residues (see Table 8 and Figure, Supplementary information 2) to perform a re-scoring step using an approach based on protein-ligand interaction networks, similar to the one recently proposed [54]. Thus, the specificity of patient 19255 used in our preliminary proof-of-concept demonstrates the capacity of the HIV-PDI resource to provide a deeper analysis with the interaction network based re-scoring when necessary.

Interestingly, if this procedure is applied to the previous 14 patients for which indinavir was ranked top compared to saquinavir (ranked between 2 and 10 according to the patient; data not shown), it appears that for all these patients, indinavir presents $40-50 \%$ more interactions than saquinavir and consequently would be the best choice for second line treatment. In other words, for these 14 patients, a treatment incorporating indinavir instead of saquinavir would have been proposed to reduce the resistance in these patients. This predicted treatment is in perfect agreement with the actual treatment given to most of the selected patients, which gave rise to an improvement of the anti-protease activity. Consequently, it appears from the present study that differences in ARV-protease interaction patterns represents a key feature to be considered when explaining loss of drug potency in patients with HIV-1 protease mutants and for predicting a suitable treatment to reduce the drug resistance observed in these patients.

\section{Conclusion}

The results of the present retrospective analysis support the robustness of the molecular modeling process carried out for building protease mutants, docking ARV, and characterizing protein-drug interaction patterns. The capacity of the HIV-PDI resource to aggregate relevant information such as critical interaction residues of diverse ARVs makes possible a more robust analysis and a satisfactory proposal for a second line of treatment. This proof-of-concept will be generalized in the future by integrating other HIV targets, especially the reverse transcriptase, and be being applied in the field for patients for whom we have full treatment histories. 
The main objective of this work is to participate in the fight against HIV resistance by developing methodologies that can associate the diverse data in the HIV-PDI database with computational methods. Our goal is to use this resource to support (i) the improvement of HIV therapeutic strategies, (ii) the selection of optimal ARV combinations, (iii) the prediction of treatment outcomes in the context of emergence and evolution of resistance-associated mutations, and (iv)Ultimately the development of new potent anti-HIV agents.

\section{Authors' contributions}

All authors have jointly developed the research concept and collaborated on the writing of the manuscript. As the main author Leo GHEMTIO has initiated the study, carried out the computational analyses, has interpreted the results, and drafted the manuscript. All authors revised the manuscript and approved its final version.

\section{Acknowledgments}

The authors thank the Bill \& Melinda Gates Foundation for financial support through the Grand Challenge Exploration grant N ${ }^{\circ} 52034$ (Round I). They are grateful to Malika Smaïl-Tabonne, Marie-Domique Devignes, Florent Petronin, and Birama Ndiaye, for their contribution to the present work.

Leo Ghemtio was supported by grants from INRIA (Institut National de Recherche en Informatique et en Automatique), CNRS (Centre National pour la Recherche Scientifique) and the Bill \& Melinda Gates Foundation.

We thank Openeye and Chemaxon for providing free software access.

This work was supported in part by Region Lorraine within the framework of the PRST MISN (MBI operation).

\section{References}

1. Dau B, Holodniy M (2009) Novel targets for antiretroviral therapy: clinical progress to date. Drugs 69: 31-50

2. Temesgen Z, Warnke D, Kasten MJ (2006) Current status of antiretrovira therapy. Expert Opin Pharmacother 7: 1541-1554.

3. Mascolini M, Larder BA, Boucher CA, Richman DD, Mellors JW (2008) Broad advances in understanding HIV resistance to antiretrovirals: report on the XVII International HIV Drug Resistance Workshop. Antivir Ther 13: 1097-1113.

4. Pillay D (2007) The priorities for antiviral drug resistance surveillance and research. J Antimicrob Chemother 60: i57-58.

5. Paar C, Palmetshofer C, Flieger K, Geit M, Kaiser R, et al. (2008) Genotypic antiretroviral resistance testing for human immunodeficiency virus type 1 integrase inhibitors by use of the TruGene sequencing system. J Clin Microbio 46: 4087-4090.

6. Shafer RW (2002) Genotypic testing for human immunodeficiency virus type 1 drug resistance. Clin Microbiol Rev 15: 247-277.

7. Garriga C, Perez-Elias MJ, Delgado R, Ruiz L, Najera R, et al. (2007) Mutational patterns and correlated amino acid substitutions in the HIV-1 protease after virological failure to nelfinavir- and lopinavir/ritonavir-based treatments. J Med Viro 79: 1617-1628.

8. Kovalevsky AY, Chumanevich AA, Liu F, Louis JM, Weber IT (2007) Caugh in the Act: the $1.5 \mathrm{~A}$ resolution crystal structures of the HIV-1 protease and the I54V mutant reveal a tetrahedral reaction intermediate. Biochemistry 46 : 14854-14864.

9. Tie Y, Kovalevsky AY, Boross P, Wang YF, Ghosh AK, (2007) Atomic resolution crystal structures of HIV-1 protease and mutants V82A and 184V with saquinavir. Proteins 67: 232-242.

10. Coles SJ, Day NE, Murray-Rust P, Rzepa HS, Zhang Y (2005) Enhancement of the chemical semantic web through the use of InChl identifiers. Org Biomo Chem 3: 1832-1834.

11. http://www.chemaxon.com/

12. Hanson R (2010) J mol - a paradigm shift in crystallographic visualization Journal of Applied Crystallography 43: 1250-1260.

13. Hong L, Zhang XC, Hartsuck JA, Tang J (2000) Crystal structure of an in vivo
HIV-1 protease mutant in complex with saquinavir: insights into the mechanisms of drug resistance. Protein Sci 9: 1898-1904

14. Lexa KW, Damm KL, Quintero JJ, Gestwicki JE, Carlson HA (2009) Clarifying allosteric control of flap conformations in the 1TW7 crystal structure of HIV-1 protease. Proteins 74:872-880

15. Perryman AL, Lin JH, McCammon JA (2004) HIV-1 protease molecula dynamics of a wild-type and of the V82F/l84V mutant: possible contributions to drug resistance and a potential new target site for drugs. Protein Sci 13 1108-1123.

16. Bennett DE, Camacho RJ, Otelea D, Kuritzkes DR, Fleury H, et al. (2009) Drug resistance mutations for surveillance of transmitted HIV-1 drug-resistance: 2009 update. PLoS One 4:e4724

17. Johnson VA, Brun-Vezinet F, Clotet B, Gunthard HF, Kuritzkes DR, et al. (2008) Update of the Drug Resistance Mutations in HIV-1. Top HIV Med 16: 138-145.

18. Boden D, Markowitz M (1998) Resistance to human immunodeficiency virus type 1 protease inhibitors. Antimicrob Agents Chemother 42: 2775-2783.

19. Kolli M, Stawiski E, Chappey C, Schiffer CA (2009) Human immunodeficiency virus type 1 protease-correlated cleavage site mutations enhance inhibitor resistance. J Virol 83:11027-11042.

20. Parera M, Fernandez G, Clotet B, Martinez MA (2007) HIV-1 protease catalytic efficiency effects caused by random single amino acid substitutions. Mol Biol Evol 24:382-387

21. Svicher V, Ceccherini-Silberstein F, Erba F, Santoro M, Gori C, et al. (2005) Novel human immunodeficiency virus type 1 protease mutations potentially involved in resistance to protease inhibitors. Antimicrob Agents Chemother 49: 2015-2025.

22. Ishima R, Louis JM (2008) A diverse view of protein dynamics from NMR studies of HIV-1 protease flaps. Proteins 70: 1408-1415.

23. Hornak V, Okur A, Rizzo RC, Simmerling C (2006) HIV-1 protease flaps spontaneously open and reclose in molecular dynamics simulations. Proc Nat Acad Sci USA 103: 915-920.

24. Wang YF, Tie Y, Boross PI, Tozser J, Ghosh AK, (2007) Potent new antivira compound shows similar inhibition and structural interactions with drug resistant mutants and wild type HIV-1 protease. J Med Chem 50: 4509-4515.

25. Seibold SA, Cukier RI (2007) A molecular dynamics study comparing a wild-type with a multiple drug resistant HIV protease: differences in flap and aspartate 25 cavity dimensions. Proteins 69: 551-565.

26. Alcaro S, Artese A, Ceccherini-Silberstein F, Ortuso F, Perno CF et al. (2009) Molecular dynamics and free energy studies on the wild-type and mutated HIV1 protease complexed with four approved drugs: mechanism of binding and drug resistance. J Chem Inf Model 49: 1751-1761.

27. Ghosh AK, Leshchenko-Yashchuk S, Anderson DD, Baldridge A, Noetze $\mathrm{M}$, et al. (2009) Design of HIV-1 protease inhibitors with pyrrolidinones and oxazolidinones as novel P1'-ligands to enhance backbone-binding interactions with protease: synthesis, biological evaluation, and protein-ligand X-ray studies. J Med Chem 52: 3902-3914.

28. Hamacher K (2008) Relating sequence evolution of HIV1-protease to its underlying molecular mechanics. Gene 422: 30-36.

29. Hou T, McLaughlin WA, Wang W (2008) Evaluating the potency of HIV-1 protease drugs to combat resistance. Proteins 71: 1163-1174.

30. Hou T, Zhang W, Wang J, Wang W (2009) Predicting drug resistance of the HIV-1 protease using molecular interaction energy components. Proteins 74 837-846

31. Jayaraman S, Shah K (2008) Comparative studies on inhibitors of HIV protease: a target for drug design. In Silico Biol 8: 427-447.

32. Jenwitheesuk E, Samudrala R (2005) Prediction of HIV-1 protease inhibito resistance using a protein-inhibitor flexible docking approach. Antivir Ther 10: 157-166.

33. Lapins M, Eklund M, Spjuth O, Prusis P, Wikberg JE (2008) Proteochemometric modeling of HIV protease susceptibility. BMC Bioinformatics 9: 181

34. Lapins M, Wikberg JE (2009) Proteochemometric modeling of drug resistance 
Citation: GHEMTIO L, SOUCHET M, DJIKENG A, KEMINSE L, KELBERT P, et al. (2011) HIV-PDI: A Protein Drug Interaction Resource for Structural Analyses of HIV Drug Resistance: 2. Examples of Use and Proof-of-Concept. J Health Med Informat 2:105. doi:10.4172/2157-7420.1000105

over the mutational space for multiple HIV protease variants and multiple protease inhibitors. J Chem Inf Model 49: 1202-1210.

35. Liu F, Kovalevsky AY, Tie Y, Ghosh AK, Harrison RW, Weber IT (2008) Effect of flap mutations on structure of HIV-1 protease and inhibition by saquinavir and darunavir. J Mol Biol 381: 102-115.

36. Meiselbach $H$, Horn $A H$, Harrer T, Sticht $H$ (2007) Insights into amprenavir resistance in E35D HIV-1 protease mutation from molecular dynamics and binding free-energy calculations. J Mol Model 13: 297-304.

37. Murphy MD, Marousek GI, Chou S (2004) HIV protease mutations associated with amprenavir resistance during salvage therapy: importance of I54M. J Clin Virol 30: 62-67.

38. Paulsen D, Elston R, Snowden W, Tisdale M, Ross L (2003) Differentiation of genotypic resistance profiles for amprenavir and lopinavir, a valuable aid for choice of therapy in protease inhibitor-experienced HIV-1-infected subjects. J Antimicrob Chemother 52: 319-323.

39. Sherman W, Tidor B (2008) Novel method for probing the specificity binding profile of ligands: applications to HIV protease. Chem Biol Drug Des 71: $387-$ 407.

40. Turner D, Schapiro JM, Brenner BG, Wainberg MA (2004) The influence of protease inhibitor resistance profiles on selection of HIV therapy in treatmentnaive patients. Antivir Ther 9: 301-314.

41. Van Marck H, Dierynck I, Kraus G, Hallenberger S, Pattery T, et al. (2009) The impact of individual human immunodeficiency virus type 1 protease mutations on drug susceptibility is highly influenced by complex interactions with the background protease sequence. J Virol 83: 9512-9520.

42. Verkhivker G (2009) Computational proteomics analysis of binding mechanisms and molecular signatures of the HIV-1 protease drugs. Artif Intell Med 45: $197-$ 206.

43. Agrafiotis DK, Bandyopadhyay D, Wegner JK, Vlijmen H (2007) Recent advances in chemoinformatics. J Chem Inf Model 47: 1279-1293.

44. Mohaar GS, Singh R, Singh V (2010) Using Chemoinformatics and Rough Set Rule Induction for HIV Drug Discovery. In Second International Conference on Machine Learning and Computing (ICMLC).
45. Almerico AM, Tutone M, Lauria A, Diana P, Barraja P, et al. (2006) A multivariate analysis of HIV-1 protease inhibitors and resistance induced by mutation. $J$ Chem Inf Model 46: 168-179.

46. Basak SC, Mills D, Garg R, Bhhatarai B (2010) A QSAR Study of HIV Protease Inhibitors Using Theoretical Descriptors. Curr Comput Aided Drug Des 6: 269282.

47. Toropov AA, Toropova AP, Benfenati E, Leszczynska D, Leszczynski J SMILES-based optimal descriptors: QSAR analysis of fullerene-based HIV-1 PR inhibitors by means of balance of correlations. J Comput Chem 31:381-392.

48. Ko GM, Reddy AS, Kumar S, Bailey BA, Garg R Computational analysis of HIV1 protease protein binding pockets. J Chem Inf Model 50: 1759-1771.

49. Wallach I, Lilien RH (2009) Prediction of sub-cavity binding preferences using an adaptive physicochemical structure representation. Bioinformatics 25: i296-i304.

50. Keiser MJ, Roth BL, Armbruster BN, Ernsberger P, Irwin JJ, et al. (2007) Relating protein pharmacology by ligand chemistry. Nat Biotechnol 25: 197206.

51. Thorsteinsdottir HB, Schwede T, Zoete V, Meuwly M (2006) How inaccuracies in protein structure models affect estimates of protein-ligand interactions: Computational analysis of HIV-I protease inhibitor binding. Proteins: Structure, Function, and Bioinformatics 65: 407-423.

52. Sadiq SK, De Fabritiis G (2010) Explicit solvent dynamics and energetics of HIV-1 protease flap opening and closing. Proteins 78: 2873-2885.

53. Warren GL, Andrews CW, Capelli AM, Clarke B, LaLonde J, et al (2006) A critical assessment of docking programs and scoring functions. J Med Chem 49: 5912-5931.

54. Xie ZR, Hwang MJ An interaction-motif-based scoring function for proteinligand docking. BMC Bioinformatics 11: 298 